\title{
Large Area Silicon Multi-Cathode Detector Developments for Microanalysis and High Speed Elemental Mapping
}

\author{
J. S. Iwanczyk*, S. Barkan*, V. D. Saveliev*, C. R. Tull*, L. Feng*, B. E. Patt*, D. E. \\ Newbury**, J. A. Small**, and N. J. Zaluzec*** \\ *Radiant Detector Technologies, 19355 Business Center Drive, Northridge CA 91324 \\ **National Institute of Standards and Technology, Gaithersburg, MD 20899 \\ ***Electron Microscopy Center, Argonne National Laboratory, Argonne IL 60439
}

We have developed a new x-ray spectrometer for electron microscopy applications, based on a $0.5 \mathrm{~cm}^{2}$ silicon multi-cathode detector (SMCD) [1]. The SMCD structure is a drifttype $\mathrm{x}$-ray detector produced from high purity silicon using state-of-the-art CMOS (complimentary metal oxide semiconductor) fabrication technology. These large area detectors possess very low capacitance and low noise at short shaping times, which allows simultaneously attaining very high count rates and excellent energy resolution required for 2-D elemental mapping [2]. In order to produce real-time 2-D elemental maps in electron beam column instruments, such as scanning electron microscopes (SEM) and transmission electron microscopes (TEM), an x-ray detector with high count rate performance $(>100 \mathrm{kcps})$, good energy resolution $(<175 \mathrm{eV}$ at $5.9 \mathrm{keV})$ and good solid angle $(\sim 0.2-0.4 \mathrm{sR})$ is desired. In addition, resistance to damage by the $300-400$ $\mathrm{keV}$ electrons in the TEM, and the ability to interface to existing data acquisition equipment, is essential.

The Vortex ${ }^{\mathrm{TM}}$ spectrometers were custom-designed to fit into the vacuum environment of an SEM at the National Institute of Standards and Technology (NIST) and into a TEM/STEM/AEM at Argonne National Laboratory (ANL). A very recent design improvement includes the complete elimination of water cooling of the spectrometer, which reduces the overall cost and improves reliability by eliminating the need for a chiller, and, very importantly, it eliminates any possible vibrations that could degrade the spatial resolution. A photograph of the new spectrometer is shown in Figure 1. The spectrometers (including the detector package interfaced with a digital pulse processor (DPP)) were first tested in the laboratory at Radiant, achieving an energy resolution of $125 \mathrm{eV} \mathrm{FHWM} \mathrm{(at} 6 \mu$ s peaking time, $5.9 \mathrm{keV}$ ) using an ${ }^{55} \mathrm{Fe}$ x-ray source, as shown in Figure 2. The count rate performance was measured as a function of input rate, and as a function of peaking time, and showed virtually no change in energy resolution, nor peak position, up to 1 Mcps.

The spectrometers were then evaluated with respect to energy resolution and count rate capability in both the SEM and TEM environments. In addition, the radiation damage resistance was evaluated in the TEM. During experiments within the electron columns, we have collected x-ray spectra from many representative specimens. The results showed excellent energy resolution of the SMCDs, as compared to traditional cryogenically cooled $\mathrm{Si}[\mathrm{Li}]$ detectors. We have been able to analyze spectra down to carbon $(277 \mathrm{eV})$, with the carbon peak fully resolved from the noise. Particular advantages can be achieved at short peaking times, where the SMCD maintained the high 
spectral resolution capabilities, unlike with $\mathrm{Si}[\mathrm{Li}]$ detectors. This feature allowed us to measure 2-D compositional maps with counting rates significantly higher than those typically used for $\mathrm{Si}[\mathrm{Li}]$ spectrometers. For example, a map of Raney nickel alloy (NiAl) was captured in only 185 seconds ( $128 \times 128$ pixels, $10 \mathrm{~ms}$ dwell per pixel with 1.3 ms overhead per pixel for beam positioning and data storage), compared to what would have taken about 8 hours with a conventional $\mathrm{Si}[\mathrm{Li}]$ spectrometer. In addition to being able to collect data clearly distinguishing the three distinct $\mathrm{Ni}-\mathrm{Al}$ phases, it is also possible to distinguish a phase containing $\mathrm{Fe}$ at the level of 0.042 mass fraction. The resistance to radiation damage was confirmed using the $300 \mathrm{keV}$ backscattered electron beam in the TEM/STEM. The spectral response was measured before, during and after electron irradiation, and showed that the detector returned to its pre-irradiation performance within $\sim 10$ minutes of removal of the high electron flux.

\section{References:}

1. S. Barkan, V. Saveliev, J. Iwanczyk, L. Feng, C. Tull, B. Patt, D. Newbury, J. Small, N. Zaluzec, "A new improved silicon multi-cathode detector (SMCD) for microanalysis and x-ray mapping applications", Microscopy Today, November 2004.

2. J. S. Iwanczyk, B.E. Patt, C.R. Tull, J.D. Segal, C.J. Kenney, J. Bradley, B. Hedman, and K.O. Hodgson, "Large Area silicon Drift Detectors for X-Rays- New Results”, IEEE Trans. Nucl. Sci. vol. 46 (1999) 284-288.

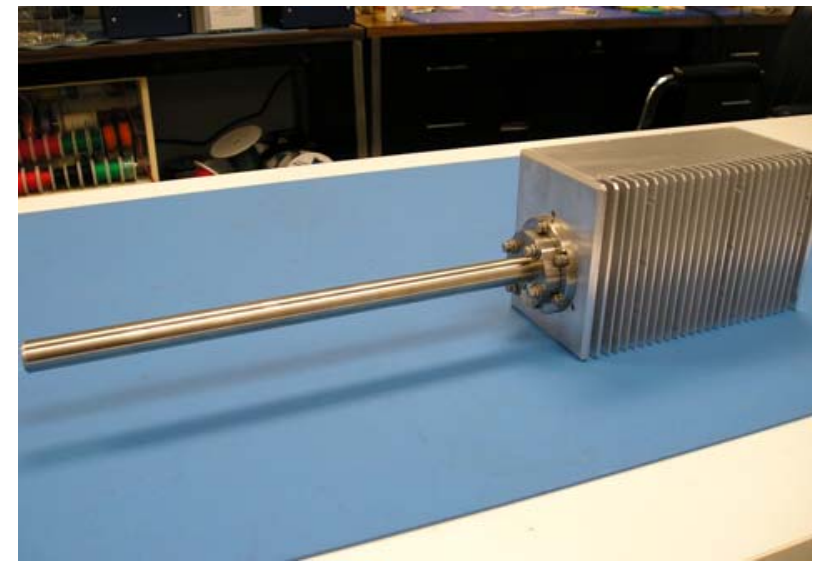

Figure 1. Photograph of the new Vortex $^{\circledR}$ EM spectrometer, which utilizes a heat pipe for heat dissipation, eliminating the need for water cooling.

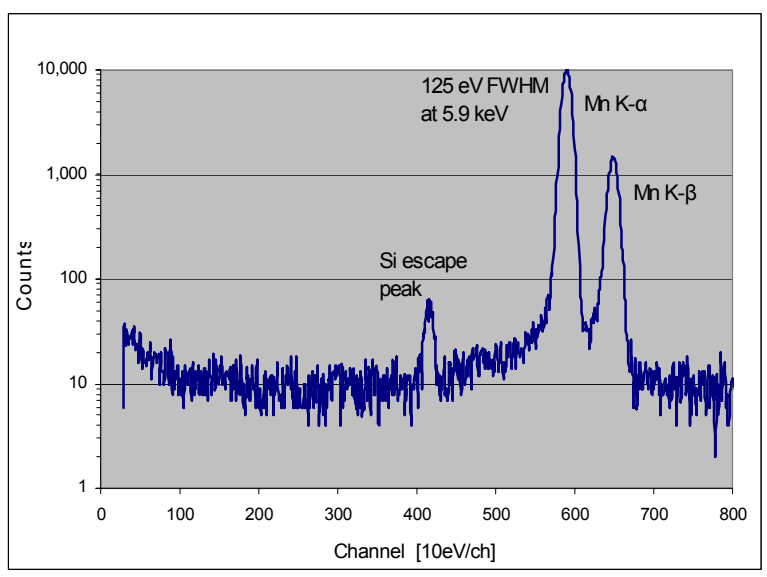

Figure 2. SMCD response to ${ }^{55} \mathrm{Fe}$, showing an energy resolution of $125 \mathrm{eV} \mathrm{FWHM}$ and peak-to-background of 2000 (at $6 \mu$ s peaking time, at $5.9 \mathrm{keV}$ ). 